\title{
Absceso de pared duodenal secundario a palillo de dientes enclavado en duodeno como causa inusual de dolor abdominal crónico
}

\section{Duodenal Wall Abscess Secondary to a Toothpick in the Duodenum is an Unusual Cause of Chronic Abdominal Pain}

Viviana Parra V., MD, ${ }^{1}$ Johana Paipilla, MD, ${ }^{1}$ Margarita Huertas Q., MD, ${ }^{2}$ Diego Aponte M., MD. ${ }^{3}$

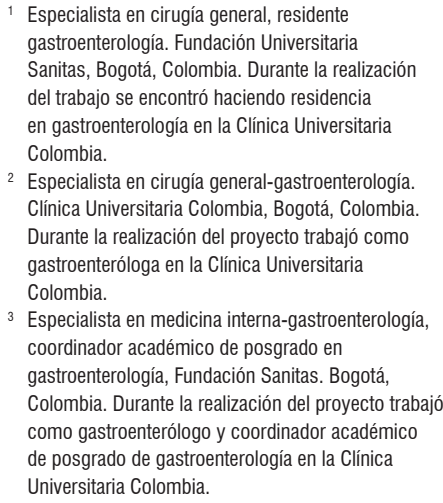

2 Especialista en cirugía general-gastroenterología. Clínica Universitaria Colombia, Bogotá, Colombia Durante la realización del proyecto trabajó como gastroenteróloga en la Clínica Universitaria Colombia.

3 Especialista en medicina interna-gastroenterología, coordinador académico de posgrado en gastroenterología, Fundación Sanitas. Bogotá, Colombia. Durante la realización del proyecto trabajó como gastroenterólogo y coordinador académico de posgrado de gastroenterología en la Clínica Universitaria Colombia.

Fecha recibido: $23-11-16$ Fecha aceptado: 21-04-17

\begin{abstract}
Resumen
Los cuerpos extraños (CE) constituyen una de las urgencias más frecuentes en gastroenterología. Aunque la mayoría de estos pasan de forma espontánea, en algunos casos pueden causar perforaciones. Presentamos el caso de un paciente masculino de 32 años quien consultó de forma ambulatoria por un cuadro de dolor abdominal de 4 meses de evolución. Dentro de los estudios solicitados se realizó una endoscopia de vías digestivas altas (EVDA) cuyo principal hallazgo fue la presencia de un cuerpo extraño (palillo de dientes) enclavado en el duodeno, que fue extraído sin complicaciones.
\end{abstract}

\section{Palabras clave}

Cuerpos extraños, palillo de dientes, perforación duodenal.

\begin{abstract}
Foreign bodies are one of the most frequent emergencies in gastroenterology. Although most of these pass spontaneously, in some cases they can cause perforations. We present the case of a 32-year-old male patient who came to the outpatient clinic after four months of abdominal pain. An endoscopy of the upper digestive tract found a toothpick embedded in the duodenum. It was extracted without complications.
\end{abstract}

Keywords

Foreign bodies, toothpicks, duodenal perforation.

\section{INTRODUCCIÓN}

La ingesta voluntaria o accidental de cuerpos extraños (CE) constituye una de las urgencias más frecuentes en el servicio de gastroenterología $(1,2)$. Tiene gran importancia dado que no solo es considerada como un reto terapéutico sino también como un reto diagnóstico por el amplio espectro de manifestaciones clínicas presentadas y el tipo de población más frecuentemente afectado (niños, pacientes con trastornos mentales, pacientes psiquiátricos y ancianos) (2).
Se considera que a pesar de que el $80 \%-90 \%$ de los CE pasan a través de todo el tracto digestivo sin causar síntomas (3-5), un 10\%-20\% requieren algún tipo de intervención médica en algún momento y un $1 \%$ de los pacientes desarrollan complicaciones graves, como abscesos de pared o perforación $(3,4)$.

En general, el palillo de dientes constituye el $9 \%$ (6) de los CE reportados en la literatura. Se considera que por su forma, tamaño y aspecto cortopunzante es un elemento que genera alta morbimortalidad por el riesgo de perforación en cualquier nivel del tracto digestivo (2). Dentro de 
los factores de riesgo para su ingesta se encuentran el abuso de alcohol, ingesta rápida de los alimentos, trastornos mentales y prótesis dentales (1). A continuación, presentamos un caso de un paciente que ingresó de forma ambulatoria para la realización de una endoscopia de vías digestivas altas (EVDA) como parte del estudio de dolor abdominal crónico en la Fundación Universitaria Clínica Colombia, a quien se le encontró un palillo de dientes enclavado en la segunda porción duodenal.

\section{CASO CLÍNICO}

Paciente masculino de 32 años sin antecedentes médicos de importancia, quien consulta de forma ambulatoria por un cuadro clínico de 4 meses de evolución de dolor abdominal intermitente predominantemente en el epigastrio. Niega otra sintomatología asociada. Dentro de los estudios practicados se realiza una tomografía de abdomen, en la que se evidencia como único hallazgo relevante el engrosamiento de la pared duodenal (Figura 1), por lo que se solicita la realización de una EVDA. El paciente ingresa el día del procedimiento en buenas condiciones generales, con signos vitales normales. En el examen físico se evidencia leve dolor a la palpación abdominal en el epigastrio sin signos de irritación peritoneal. Se realiza la EVDA, en la que se evidencia un cuerpo extraño (palillo de dientes) enclavado en la pared anteroposterior de la segunda porción del duodeno (Figura 2). Posterior a la extracción del palillo con una pinza de cuerpo extraño (Figura 3) se evidencia la salida de material purulento a través de los orificios sin evidencia clara de perforación (Figura 4).

Dados los hallazgos, el paciente es hospitalizado para observación, inicio de antibioticoterapia y realización de una nueva tomografía abdominal para descartar una perforación duodenal. El paciente presenta mejoría completa de su sintomatología a las 24 horas de su ingreso y es dado de alta una vez se confirma la ausencia de perforación duodenal en la tomografía realizada (Figura 5). En el interrogatorio realizado, el paciente no recuerda haber ingerido un palillo de dientes previo al inicio de su sintomatología.

\section{DISCUSION}

En la literatura existen numerosos reportes de manejo de $\mathrm{CE}$ en el tracto digestivo, debido a que es un motivo de consulta frecuente en el servicio de gastroenterología; sin embargo, complicaciones como perforación o abscesos solo alcanzan el $1 \%(3,4)$ de los casos.

En general, el grupo etario con mayor riesgo de ingesta de CE es la población infantil, que representa el 60\%-80\% (7) de los casos; aunque, en los adultos, la incidencia es alta en pacientes con trastornos mentales, presos, alcohólicos y ancianos (2). En un estudio realizado entre el 2005 y 2007 en el Hospital Cardiovascular de Soacha, se encontró que

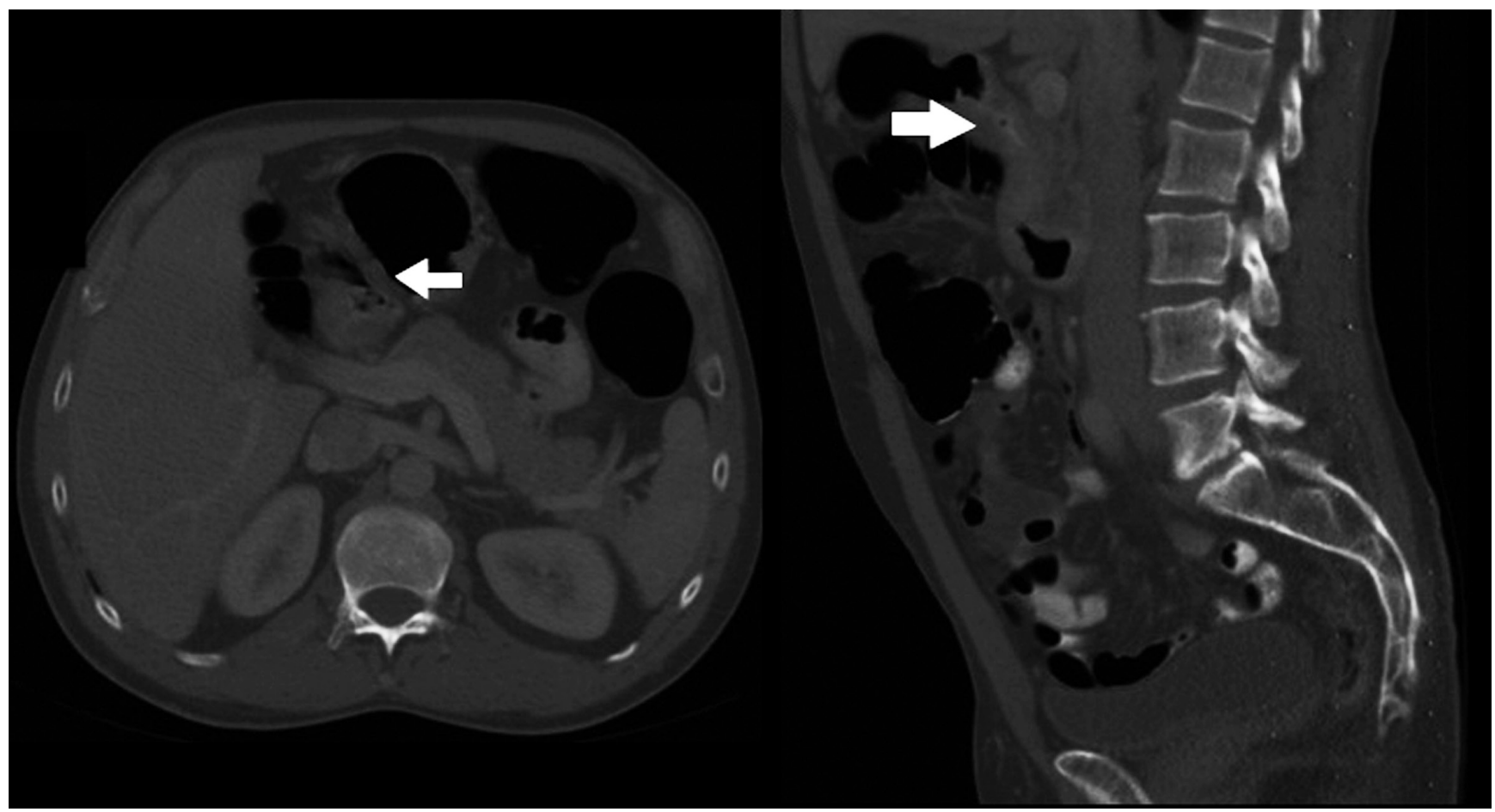

Figura 1. Imagen tomográfica engrosamiento de pared duodenal. 


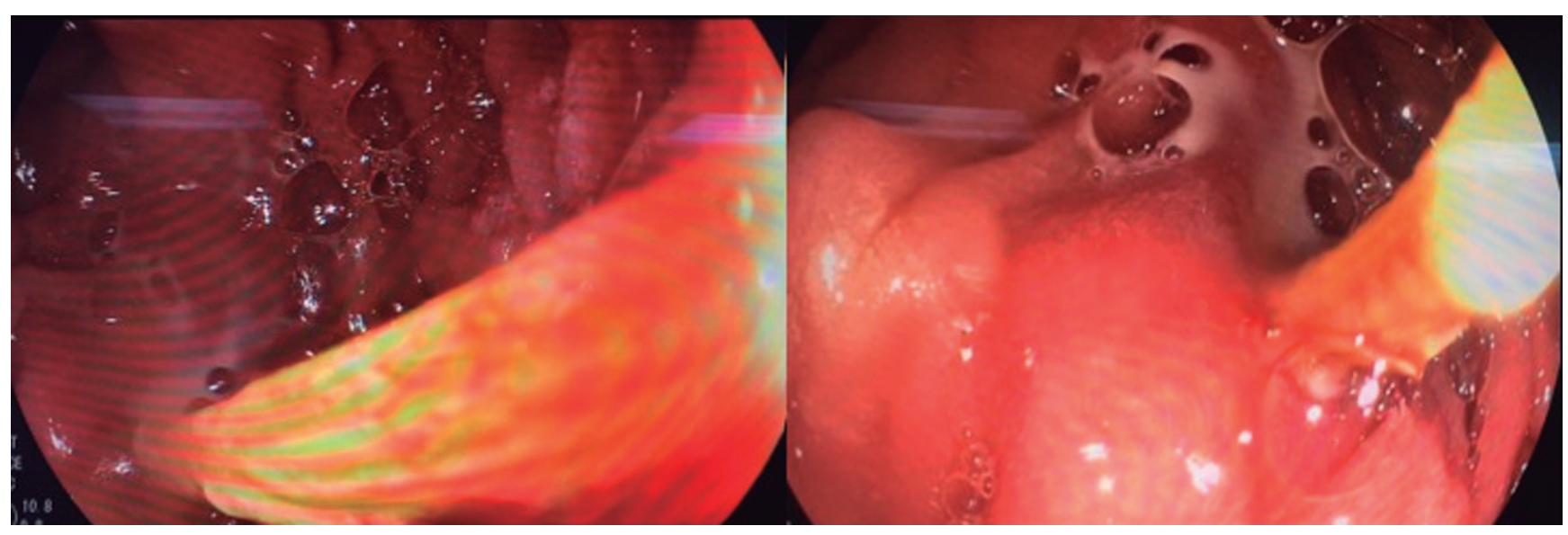

Figura 2. Imagen endoscópica de cuerpo extraño (palillo) enclavado en la pared de la segunda porción duodenal con presencia de pus a través del orificio de la pared anterior.

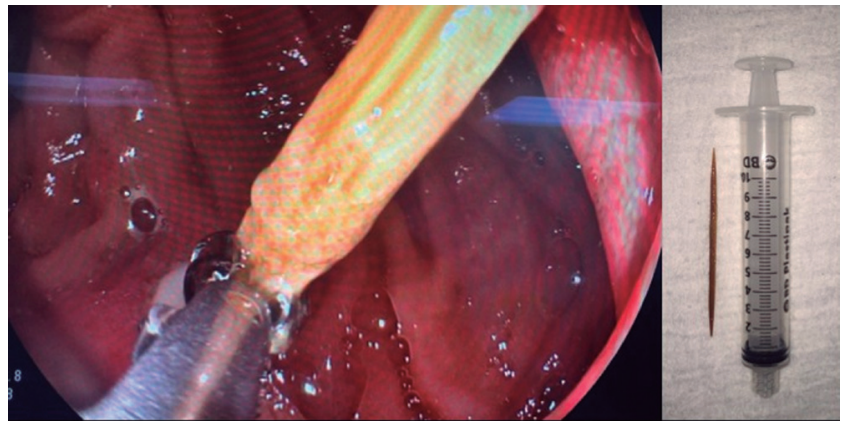

Figura 3. Imagen endoscópica de extracción de palillo con pinza de cuerpo extraño.

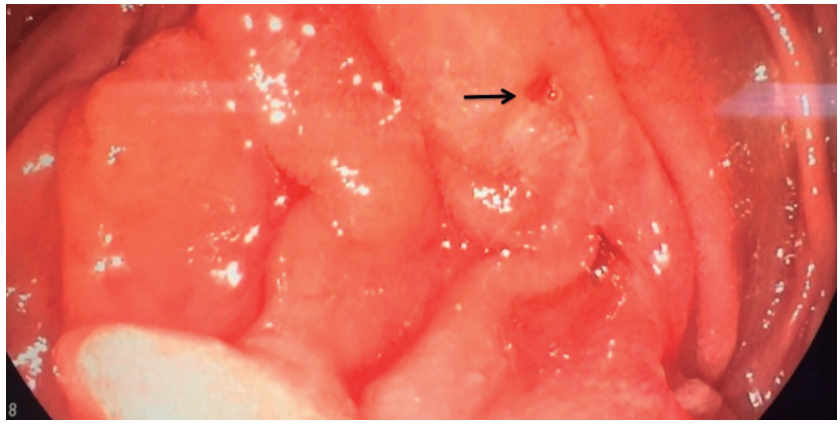

Figura 4. Imagen endoscópica de pared duodenal posterior a extracción de cuerpo extraño sin evidencia clara de perforación.

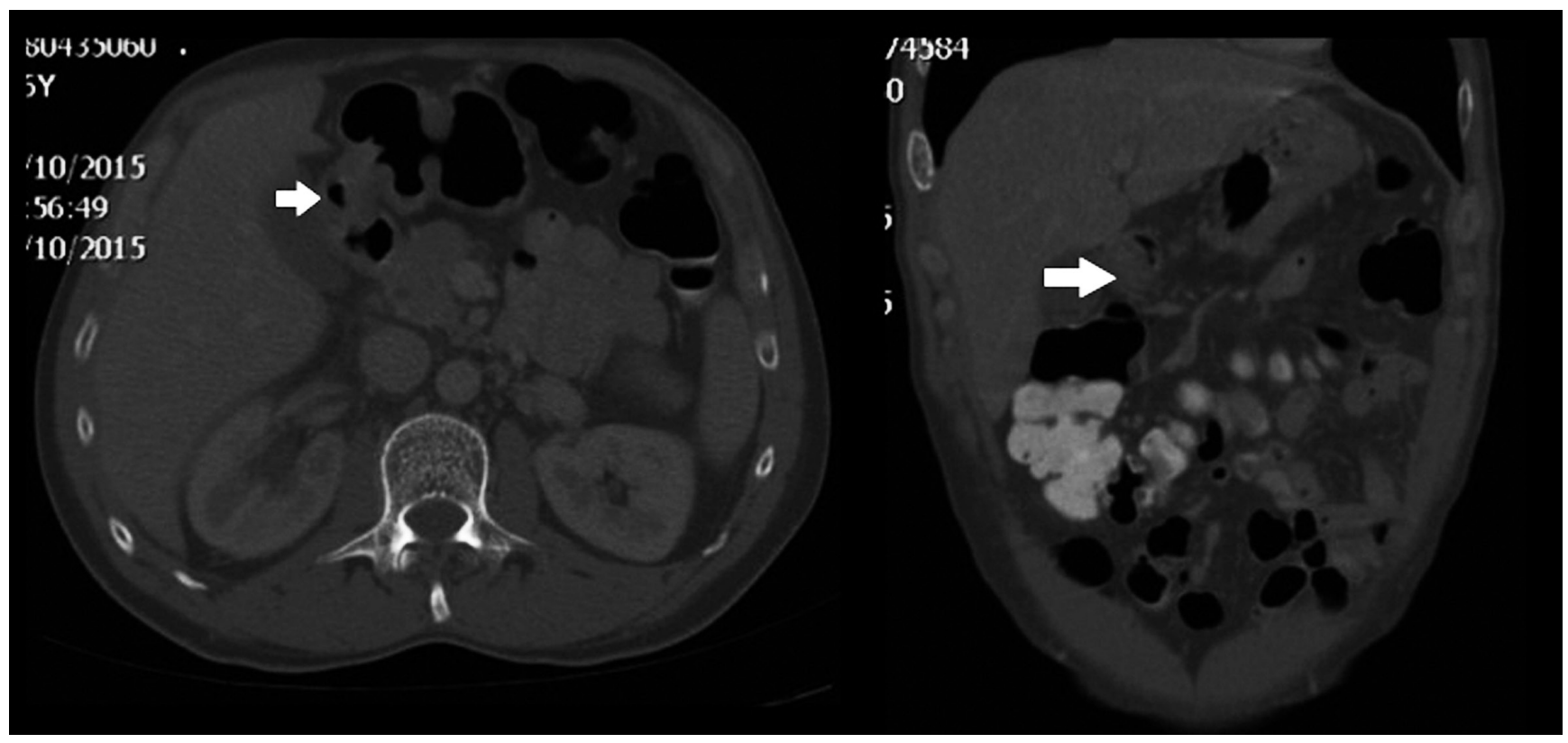

Figura 5. Imagen tomográfica posterior a extracción de cuerpo extraño en la que se descarta perforación. 
las monedas, las partes de juguetes y las pilas de reloj fueron en este orden los cuerpos extraños más frecuentemente ingeridos por los niños. Asimismo, describió que este motivo de consulta es la segunda indicación más frecuente de realización de EVDA de urgencias en niños después de la hemorragia digestiva y que la etapa preescolar (menores de 5 años) es el grupo etario con mayor incidencia (7).

Cualquier $\mathrm{CE}$ es posible, siendo más frecuentes las monedas, el bolo alimenticio impactado y huesos de pollo o espinas de pescado $(8,9)$. La ingesta de un palillo de dientes es rara, constituye en general el $9 \%$ de los casos (6); pero por su carácter cortopunzante y su tamaño se han documentado diferentes tipos de complicaciones secundarias a su perforación en el tracto gastrointestinal (TGI), incluyendo desde el alojamiento en pleura, hígado, pericardio, peritoneo, riñón, uréter y vejiga (10-15) hasta complicaciones más graves, como fistulas del duodeno con la aorta o la vena cava inferior (16-18).

En cuanto al diagnóstico, es importante tener en cuenta una historia clínica completa; sin embargo, en muchas ocasiones es difícil identificar con certeza si hubo ingesta o no de un $\mathrm{CE}$, como es el caso de nuestro paciente, quien no recordaba haber ingerido el palillo de dientes y cuyo cuadro clínico fue muy bizarro, lo que dificultó la realización del diagnóstico. En una revisión de la literatura realizada en el 2013 por LeeWon Chong (8) se encontró que, en la población adulta, los hombres presentan una mayor incidencia de ingesta de CE (73\%) que las mujeres (27\%), con una edad media de 50,4 \pm 18,6 años. Por otro lado, se identificó la amplia e inespecífica variedad de síntomas que pueden presentarse secundarios a la perforación del TGI por un CE: dolor abdominal $(77,3 \%)$, fiebre (58\%), vómito (19,3\%) y náuseas (13,6\%).

En la presentación aguda, cuando existe una alta sospecha de ingesta de CE son de gran utilidad las imágenes diagnósticas. Se considera que la radiografía convencional puede visualizar, según las series descritas, hasta un $65 \%$ de CE radioopacos (1). La limitación de este estudio se debe a que su rendimiento depende de la radiolucidez del CE; así, los palillos ofrecen una dificultad adicional ya que solo entre 5\%-15\% son detectables en radiografías $(2,19)$. La tomografía confiere una mayor utilidad en casos específicos dado que puede descartar complicaciones como las perforaciones y abscesos. Adicionalmente, puede brindar mejor información sobre la localización del CE (intra o extraluminal) con el fin de determinar la conducta y, en caso de requerirlo, la vía de abordaje $(20,21)$.

En algunos casos donde no se realizan previamente imágenes diagnósticas o cuando la sospecha de $\mathrm{CE}$ es muy alta, el examen de elección es la EVDA, dado que no solo es útil para realizar el diagnóstico sino también permite la extracción del CE en la mayoría de los casos $(2,3)$. Por último, algunos pacientes son diagnosticados y manejados de forma intraoperatoria cuando ninguno de los elementos previamente nombrados brindan la información suficiente y por el estado del paciente se amerita llevarlo a cirugía $(9,10)$. En el caso de nuestro paciente, el diagnóstico fue realizado de forma endoscópica, dado que en la imagen tomográfica únicamente se observó engrosamiento de la pared duodenal.

El tratamiento de perforaciones secundarias a CE debe ser individualizado dependiendo de la edad, el CE ingerido, los hallazgos imagenológicos y, principalmente, del estado del paciente al momento del diagnóstico. Como en nuestro paciente, cada vez hay más reportes de casos que muestran cómo el manejo conservador (suspender la vía oral, suministrar nutrición enteral $[\mathrm{NE}]$ según la necesidad, realizar un cubrimiento con antibióticos de amplio espectro y continuar con la vigilancia clínica del paciente) es una excelente herramienta una vez se realiza la extracción endoscópica del CE en pacientes estables hemodinámicamente, que no muestren signos clínicos que indiquen necesidad de una intervención quirúrgica de forma urgente (abdomen agudo y/o signos de respuesta inflamatoria sistémica) $(2,4)$. Finalmente, cabe mencionar la importancia hoy en día del manejo endoscópico de perforaciones intestinales, dada su alta tasa de éxito técnico y clínico a largo plazo. Herramientas como: clips metálicos, que son principalmente útiles en perforaciones menores de $2 \mathrm{~cm}$, el sistema OVESCO u OTCS (acrónimo del inglés, over the scope clip) que puede llegar a ser exitoso en perforaciones hasta de $3 \mathrm{~cm}$ y, en casos muy esporádicos, colocación de prótesis metálica totalmente cubierta. Todos estos elementos han disminuido sustancialmente la necesidad de procedimientos quirúrgicos y las tasas de morbimortalidad (5-7).

\section{REFERENCIAS}

1. Steinbach C, Stockmann M, Jara M, et al. Accidentally ingested toothpicks causing severe gastrointestinal injury: a practical guideline for diagnosis and therapy based on 136 case reports. World J Surg. 2014;38(2):371-7.

2. Mark D, Ferris K, Martel G, et al. Radiological diagnosis of a small bowel perforation secondary to toothpick ingestion. BMJ Case Rep. 2013;2013. pii: bcr20130098693.

3. Chong L-W. Successful treatment of liver abscess secondary to foreign body penetration of the alimentary tract: A case report and literature review. World J Gastroenterol. 2014;20(13):3703-11. Available from: http://www.pubmedcentral.nih.gov/articlerender.fcgi?artid=3974541\&too $1=$ pmcentrez\&rendertype $=$ abstract

4. Zouros E, Oikonomou D, Theoharis G, et al. Perforation of the cecum by a toothpick: report of a case and review of the literature. J Emerg Med. 2014;47(6):e133-7. Available from: http://www.ncbi.nlm.nih.gov/pubmed/25300206

5. Robert B, Bartoli E, Fumery M, et al. Duodenal perforation due to toothpick perforation, an uncommon cause of chronic abdominal pain. Endoscopy. 2012;44 Suppl 2 
UCTN:E27-8. Available from: http://www.ncbi.nlm.nih. gov/pubmed/22396261

6. Tenner S, Wong RC, Carr-Locke D, et al. Toothpick ingestion as a cause of acute and chronic duodenal inflammation. Am J Gastroenterol. 1996;91(9):1860-2. Available from: http://www.ncbi.nlm.nih.gov/pubmed/8792719

7. Ospina J. Cuerpos extraños en tracto gastrointestinal en niños. Rev Col Gastroenterol. 2008;23(3):233-8. Available from: http://www.scielo.org.co/pdf/rcg/v23n3/v23n3a07.pdf

8. Santos SA, Alberto SCF, Cruz E, et al. Hepatic abscess induced by foreign body: case report and literature review. World J Gastroenterol. 2007;13(9):1466-70. Available from: http://www.pubmedcentral.nih.gov/articlerender.fcgi?arti $\mathrm{d}=4146938$ \&tool $=$ pmcentrez\&rendertype $=$ abstract

9. Ricci S, Massoni F, Schiffino L, et al. Foreign bodies ingestion: What responsibility? J Forensic Leg Med. 2014;23:58. Available from: http://www.ncbi.nlm.nih.gov/pub$\mathrm{med} / 24661696$

10. Li SF, Ender K. Toothpick injury mimicking renal colic: case report and systematic review. J Emerg Med. 2002;23(1):358. Available from: http://www.ncbi.nlm.nih.gov/pubmed/12217469

11. König PS, Kronenberg F, Joannidis M, et al. Recurrent abdominal pain caused by a toothpick in a CAPD patient. Adv Perit Dial. 1991;7:96. Available from: http://www.ncbi. nlm.nih.gov/pubmed/1680466

12. O’Dea MJ, Malek RS. Foreign body in bladder and perivesicular inflammation masquerading as pelvic lipomatosis. J Urol. 1976;116(5):669-70. Available from: http://www. ncbi.nlm.nih.gov/pubmed/789923

13. Alagiri M, Rabinovitch $\mathrm{HH}$. Toothpick migration into bladder presents as abdominal pain and hematuria. Urology.
1998;52(6):1130-1. Available from: http://www.ncbi.nlm. nih.gov/pubmed/9836569

14. Schwartz JT, Graham DY. Toothpick perforation of the intestines. Ann Surg. 1977;185(1):64-6. Available from: http://www.pubmedcentral.nih.gov/articlerender.fcgi?arti $\mathrm{d}=1396248$ \&tool $=$ pmcentrez\&rendertype $=$ abstract

15. Plavcan WG, McWilliams WA. Toothpick obstruction of the ureter. J Urol. 1988;139(1):114-5. Available from: http:// www.ncbi.nlm.nih.gov/pubmed/3275793

16. Justiniani FR, Wigoda L, Ortega RS. Duodenocaval fistula due to toothpick perforation. JAMA. 1974;227(7):788-9. Available from: http://www.ncbi.nlm.nih.gov/pubmed/4405846

17. Dicicco BS, Heit HA, Peterson JE, et al. Massive bleeding due to arterial-enteric fistula from an ingested toothpick. J Clin Gastroenterol. 1985;7(4):292-5. Available from: http://www.ncbi.nlm.nih.gov/pubmed/3876367

18. Fry D, Flint LM, Richardson JD. Aorticoduodenal fistula secondary to a toothpick.J Ky Med Assoc. 1978;76(9):441. Available from: http://www.ncbi.nlm.nih.gov/pubmed/308975

19. Anderson MA, Newmeyer WL, Kilgore ES. Diagnosis and treatment of retained foreign bodies in the hand. Am J Surg. 1982;144(1):63-7. Available from: http://www.ncbi.nlm. nih.gov/pubmed/7091533

20. Lee JH, Kim HC, Yang DM, et al. What is the role of plain radiography in patients with foreign bodies in the gastrointestinal tract? Clin Imaging;36(5):447-54. Available from: http://www.ncbi.nlm.nih.gov/pubmed/22920344

21. Coulier B, Tancredi M-H, Ramboux A. Spiral CT and multidetector-row CT diagnosis of perforation of the small intestine caused by ingested foreign bodies. Eur Radiol. 2004;14(10):1918-25. Available from: http://www.ncbi. nlm.nih.gov/pubmed/15378256 